\title{
LA EXPERIENCIA DE LOS PROCESOS DE AUTOEVALUACIÓN Y ACREDITACIÓN DE LOS INSTITUTOS DE EDUCACIÓN SUPERIOR TECNOLÓGICA
}

\author{
EXPERIENCE ON SELF-ASSESSMENT AND ACCREDITATION PROCESSES \\ OF THE SUPERIOR TECHNOLOGICAL INSTITUTES
}

Mónica Escalante ${ }^{1}$

\section{RESUMEN}

Los procesos de autoevaluación y acreditación de los institutos de educación superior tecnológica (IEST) conducidos por el CONEACES fueron respuesta a una necesidad imperante de promover una cultura de calidad. El propósito fue movilizar procesos de cambio que partiesen de una evaluación interna, autoevaluación orientada a la autorregulación y mejora continua, y se dirigiesen a una externa, imparcial y objetiva de su cumplimiento Tuvo como estrategia el trabajo participativo y como fortaleza la capacidad de convocatoria necesaria para involucrar a todos los actores relacionados con los IEST: autoridades, promotores, directores, docentes, especialistas, funcionarios, académicos, expertos nacionales e internacionales. Evidencia del trabajo realizado han sido los más de 200 IEST que hasta finales de 2014 se encontraban en proceso de autoevaluación, evaluación externa o ya se han acreditado.

\section{Palabras clave}

Procesos, Autoevaluación y acreditación, Institutos de Educación Superior Tecnológica

\section{ABSTRACT}

The self-assessment and accreditation processes of the superior technological educational institutes (IEST) conducted by the Council of Assessment, Accreditation and Certification of Quality of Nonuniversity Superior Education (CONEACES) served as a response to a prevailing necessity to promote a culture of quality. The objective was to mobilize change processes that start from an internal assessment, a self-assessment guided to the self-regulation and constant improvement, and that can be directed to an external, impartial and objective fulfillment. The survey had the participatory work as a strategy and the rallying ability as a strength necessary to involve all of the actors related to the IEST: authorities, promoters, directors, teachers, staff members, academicians, national and international experts. Evidence to the study has been more than the 200 IEST that until the end of the year 2014 were in self-assessment process, third party assessment or have already been accredited.

\section{Keywords}

Process, self-assessment, accreditation, Superior Technological Institutes

\footnotetext{
Lic. En Educación Secundaria en la especialidad de Lengua y Literatura (UNIFÉ). Magíster en Educación con mención en Tecnología Educativa (UNIFÉ), en TIC aplicadas a la Educación (Universidad de Salamanca). Profesora Principal de la Facultad de Ciencias de la Educación de la UNIFÉ, docente de la Universidad Peruana Cayetano Heredia y consultora en tema educativos. Sus publicaciones han estado relacionadas con temas de salud y educación.
} 
El presente artículo tiene como propósito presentar de manera analíticas los procesos $y$ acciones desarrollados por CONEACES para que los institutos de educación superior tecnológica (IEST $=$ puedan ofrecer un servicio de calidad que contribuya a articular el sector educación con el mundo del trabajo. Para ello, se describe el contexto en el que se dio inicio a los procesos de evaluación y acreditación con la intención de comprender las necesidades y prioridades de la educación superior. Asimismo, se explica con detalle el proceso de diseño, implementación y validación de los estándares y criterios de evaluación para la acreditación de las carreras profesionales de los institutos y escuelas de educación superior tecnológicos y escuelas de formación técnico profesional de los sectores Defensa e Interior. Finalmente, se describe como los procesos de evaluación y acreditación se han desarrollado hasta fines de 2014. En este análisis no se incluye la experiencia de las instituciones de formación docente (IFD) ni de las instituciones de salud.

\section{IDENTIFICACIÓN DEL CONTEXTO PARA EL DISEÑO, ELABORACIÓN $Y$ VALIDACIÓN DE LOS ESTÁNDARES DE LOS INSTITUTOS DE EDUCACIÓN SUPERIOR TECNOLÓGICA}

Al inicio del presente siglo, la necesidad de ordenary articular la diversidad de instituciones existentes fuera de los patrones universitarios se hizo necesaria. La oferta desregulada de instituciones de educación superior tecnológica impulsó la toma de decisiones orientadas a la regulación y caracterización de ese sector educativo.

En los IEST se observó un significativo incremento en el número de matrículas y de instituciones de baja calidad carentes de aprobación para su funcionamiento. En consecuencia, estas instituciones fueron cuestionadas por la incoherencia entre el alto número de proveedores y los bajos niveles de calidad detectados en su oferta educativa (MINEDU y CNED, 2007; De la Haya, 2005).
Con respecto a la implementación del sistema de aseguramiento de la calidad, el subsistema de educación superior no universitaria estuvo dinamizado durante el periodo 1990-2010 por los proyectos impulsados desde el MINEDU y el Ministerio de Trabajo. Para ello se contó con el apoyo de la cooperación internacional (AECI, UE, BID). Se constituyeron un total de ocho proyectos de mejoramiento de la calidad y fortalecimiento de la formación profesional no universitaria. (SINEACE, 2011)

Los proyectos se desarrollaron de manera independiente, pero no mostraron incidencia sobre las políticas del Estado. A continuación, se describen algunos logros: (SINEACE 2011)

- Diagnóstico general de la oferta de educación técnica y ocupacional del país (MINEDU/GRADE).

- Se diseñó una propuesta de "subsistema de formación profesional técnica, articulado al sistema educativo y vinculado al mundo del trabajo" a partir del proyecto "Diseño del Sistema de Formación Profesional Técnica en el Perú" (MINEDU-AECI).

- Catálogo Nacional de Títulos y Certificaciones, el cual organizó la oferta formativa en función a 20 familias profesionales, con el objetivo de ser un referente flexible de los requerimientos de la economía nacional.

El catálogo representó un esfuerzo significativo por responder a las demandas del sector productivo. Se definieron los perfiles y las competencias profesionales de cada especialidad con la participación de expertos del sector productivo. Se deseaba era desarrollar en los estudiantes las competencias que les permitieran insertarse en el mercado laboral de manera exitosa. La fase experimental del Catálogo concluyó en 2008 y en 2009 se inició su generalización. Sin embargo, este trabajo no se ajustó en un $100 \%$ al modelo de sistema de educación técnica y formación profesional propuestos.

Es importante acotar que ya en 2006 se habían publicado los Lineamientos Nacionales 
de Política de la Formación Profesional mediante el Decreto Supremo ( $\mathrm{N}^{\circ}$ 021-2006). Esta norma incluyó políticas y estrategias de formación profesional técnica y constituyó un referente para la formulación de una política nacional de formación profesional. Los lineamientos consideraron: el diálogo social, la concertación y la negociación; la calidad y pertinencia de la formación profesional; la información del mercado laboral y formativo; y la sostenibilidad, cuya finalidad estuvo orientada a:

Mejorar la calidad de la formación profesional en el Perú, sobre la base del fortalecimiento de los espacios de dialogo social entre los actores; de la calidad y pertinencia de la formación profesional; de la creación, intercambio $y$ sistematización oportuna de información sobre el mercado laboral y formativo; y, de la sostenibilidad de las políticas de formación profesional y promoción del empleo. (SINEACE, 2011)

A pesar de los muchos esfuerzos realizados, se sigue observando un servicio heterogéneo y una oferta de baja calidad, lo que se videncia en una difícil inserción laboral. Entre las causas de esta situación se puede mencionar: (Jaramillo, Valdivia y Valenzuela, 2007 y Ángeles, et al., 2012)

- La falta de continuidad de las políticas en el campo de la formación profesional $y$ el desarrollo de capacidades como consecuencia de la débil institucionalidad del sector.

- El equipo técnico responsable, no ha presentado un enfoque claro de formación profesional que implemente los lineamientos de política de formación profesional del Decreto Supremo N. ${ }^{\circ} 021-2006$.

- No existe un trabajo articulado entre los sectores educación, trabajo promoción social, lo que redunda en una capacidad limitada para concretar políticas multisectoriales que vinculen -efectivamente- la formación no universitaria con el mercado laboral.
Sin embargo, se debe destacar la participación de la cooperación internacional, la cual ha sido determinante para promover una formación basada en el desarrollo de habilidades. Gracias a ello, se establecieron políticas piloto, pero luego no fueron implementadas. Ejemplo de estos Programas: (Instituto Peruano de Evaluación, Acreditación y Certificación de la Calidad de la Educación Básica [IPEBAȘ, 2011)

- Programa Marco de Formación Profesional Tecnológica y Pedagógica en Perú (FORTE-PE) ejecutado entre 1998 y 2003. El Programa comprendió dos proyectos: Proyecto Horizontal de Formación de Formadores de Educación Tecnológica (PROTEC), y Proyecto de Formación de Maestros en Educación Bilingüe Intercultural de la Amazonia Peruana (PROEBI).

- Programa de Apoyo al Ajuste Social Estructural (PASE) se inició en 1997 con la intención de permitir el ingreso al mundo laboral en corto plazo a jóvenes de áreas rurales

- Programa Piloto de Formación Profesional Técnica (MECEP-BID) se desarrollo entre 2001 y 2005. Tuvo como propósito apoyar el desarrollo de modelos de gestión que vincularan a los centros de formación profesional técnica con las empresas.

- Apoyo a la Formación para la Inserción Laboral en el Perú II (APROLAB II) se ejecutó durante el periodo 2006-2011. Tuvo como objetivo general contribuir a la mayor competitividad y calidad de la fuerza laboral para el desarrollo económico y la reducción de la pobreza en el Perú.

Asimismo, instituciones peruanas, como el CNED, han propuesto articular toda la educación superior para ofrecer un aprendizaje modular y fluido para que los estudiantes puedan avanzar en su educación, sin que la modalidad adoptada al inicio sea una barrera.

Finalmente, si ha habido avances significativos, aún queda mucho por hacer 
para garantizar la calidad de la educación superior no universitaria, como por ejemplo: (SINEACE, 2011)

- Articular la oferta formativa y la demanda de los perfiles de profesionales desde el mundo laboral.

- Articular y generar sinergias entre la educación superior universitaria y no universitaria.

- Ofrecer información oficial a los estudiantes acerca de la calidad de la oferta de formación.

- Garantizar información confiable acerca de los indicadores del sistema de aseguramiento de la calidad.

Como se puede inferir, si bien las políticas orientadas a mejorar la calidad de la educación superior no universitaria ofrecidas en los IEST no han sido articuladas ni sostenidas, se observó que sí han estado presentes en las agendas de los diferentes gobiernos. Además, se evidencian avances significativos con respecto a la organización de la diversidad de alternativas que se ofrecen: las careras están organizadas en familias profesionales. Todo lo actuado hasta la fecha por iniciativa del gobierno o de organismos internacionales ha contribuido a preparar el contexto para que los procesos de evaluación y acreditación se instalen $y$ desarrollen en un ambiente democrático, favorable y de participación conjunta: autoridades y comunidad educativa. Los retos son muchos, pero existe la voluntad de trabajar por mejorar la calidad de los IEST.

Sin embargo, algunos IEST de vanguardia ya habían iniciado procesos de evaluación conducentes a la acreditación, ya que eran conscientes de la necesidades de ser reconocidos como instituciones de excelencia. Las primeras experiencias en procesos de evaluación y acreditación en los IEST en el Perú se iniciaron a principios de la primera década del siglo XXI. Los IEST que tomaron la decisión con la intención de garantizar la calidad del servicio que ofrecían fueron: Instituto Tecnológico Superior TECSUP e Instituto Superior Tecnológico CIBERTEC. Para ello eligieron acreditadoras extranjeras: Accreditation Board for Engineering and Technology (ABBET) y Agencia Alemana que Acredita los Programas de Ingeniería (ASIIN)

\section{A) Caso TECSUP}

- TECSUP inició su proceso de acreditación en el año 2003 y en 2006 logró su primera carrera acreditada: Electrónica y Automatización Industrial. Al 2012 ha logrado acreditar todas sus carreras tanto en Lima como en Arequipa. Como se observa en la Tabla 1

Tabla 1

Carreras de TECSUP Acreditadas por ABET

\begin{tabular}{|l|l|}
\hline \multicolumn{1}{|c|}{ TECSUP Arequipa } & \multicolumn{1}{c|}{ TECSUP Lima } \\
\hline ○ Mantenimiento de Maquinaria de Planta & ○ Procesos Químicos y Metalúrgicos \\
○ Electrotecnia Industrial & ○ Mantenimiento de Maquinaria de Planta \\
○ Redes y Comunicaciones de Datos & ○ Electrotecnia Industrial \\
& ○ Electrónica y Automatización Industrial \\
& ○ Redes y Comunicaciones de Datos \\
\hline
\end{tabular}

ABBET es una agencia de los Estados Unidos que acredita carreras o programas en Ingeniería y Tecnología. Esta Agencia ha acreditado 3100 carreras en casi 700 instituciones de educación superior en 24 países. TECSUP fue la primera institución educativa en su tipo en el Perú en recibir el reconocimiento de ABET. "La acreditación $\mathrm{ABET}$ es la garantía o aseguramiento de que el programa acreditado cumple con los estándares de calidad establecidos para la profesión en la que prepara a sus estudiantes." (TECSUP, 2014, ๆ. 2) 
Tabla 2

Carreras de TECSUP acreditadas por ASIIN

\begin{tabular}{|l|l|}
\hline \multicolumn{1}{|c|}{ TECSUP Arequipa } & \multicolumn{1}{c|}{ TECSUP Lima } \\
\hline ○ Mantenimiento de Maquinaria de Planta & ○ Procesos Químicos y Metalúrgicos \\
○ Mantenimiento de Maquinaria Pesada & ○ Mantenimiento de Maquinaria de Planta \\
○ Electrotecnia Industrial & ○ Mantenimiento de Maquinaria Pesada \\
& ○ Electrotecnia Industrial \\
& ○ Electrónica y Automatización Industrial \\
\hline
\end{tabular}

ASIIN, como se muestra en la Tabla 2 , ha brindado a las carreras de TECSUP la acreditación como programas de ingeniería más orientados a la práctica. European Network for Accreditation of Engineering Education (ENAEE) reconoce la calidad y el nivel de estas como equivalentes a las especialidades de ingeniería correspondientes al pre grado en el sistema europeo. ASIIN otorgó el sello EURACE que facilitó la posibilidad de estudiar programas de maestría en Europa, así como, trabajar en los 13 países miembros (Alemania, Inglaterra, Italia, Francia, Irlanda, Dinamarca, Suiza, Rusia, Portugal, Bélgica, Rumania, Turquía, Austria).

\section{B. Caso CIBERTEC}

CIBERTEC desarrolló su proceso de evaluación 2009-2010 y en setiembre de este último año recibió la acreditación de ABBET en la carrera de Computación e Informática.

Varios países ya contaban con agencias acreditadoras nacionales para certificar las carreras que ofrecían. En el Perú se contaba con un único sistema de acreditación legalmente instituido: la Comisión para Acreditación de Facultades o Escuelas de Medicina CAFME (Ley N. ${ }^{\circ}$ 27154).

Una iniciativa privada fue la que tuvo el Instituto de la Calidad en la Acreditación de Carreras Profesionales de Ingeniería y Tecnología (ICACIT). El ICACIT firmó un memorando de entendimiento con ABET para promover la colaboración en el proyecto piloto de acreditación de carreras de ingeniería y tecnología. El ICACIT conformado inicialmente por la Universidad Nacional de Ingeniería, la Pontificia Universidad Católica del Perú, la Universidad San Martín de Porres, la Universidad Peruana de Ciencias Aplicadas y el Instituto Tecnológico Superior TECSUP incorporó luego a otras instituciones como la Universidad Nacional del Callao, la Universidad Ricardo Palma, la Universidad Tecnológica del Perú, la Universidad Privada del Norte y CIBERTEC. El Instituto cuenta ya con un amplio respaldo de asociaciones de industriales como la Confederación Nacional de Instituciones Empresariales Privadas (CONFIEP), el Instituto de Ingenieros Eléctricos y Electrónicos (IEEE), el Colegio de Ingenieros del Perú (CIP), la Asociación Electrotécnica Peruana (AEP), la Academia Peruana de Ingeniería (API) y la Asociación Peruana de Productores de Software (APESOFT).

\section{DISEÑO, IMPLEMENTACIÓN Y VALIDACIÓN DEL PROCESO DE EVALUACIÓN Y ACREDITACIÓN}

Con respecto a la elaboración y validación de los estándares de IEST propuesto por el CONEACES, este se sustentó en los lineamientos que articularon lo teóricoconceptual, lo metodológico-operativo del los estándares de los IFD. La transferencia fue realizada luego de un proceso de consultas y experiencias de validación con la participación de los actores directos $e$ indirectos. El fin era generar los mecanismos que facilitaran la implementación de los procesos de evaluación y acreditación de las instituciones de formación no universitaria. No se debe olvidar que instituciones como TECSUP, CIBERTEC ya tenían experiencia con acreditadoras extranjeras $y$ participaron con la mejor disposición en esta primera etapa. 
La implementación de los procesos de evaluación y acreditación implicaron la participación de los miembros de CONEACES, directivos, docentes y grupos de interés de los IEST. Se contó con el apoyo político y el interés de las propias instituciones. En 2009 se inició la implementación de los procesos, contando como experiencia previa lo trabajado en los IFD.

\subsection{Construcción y validación de estándares y manuales}

CONEACES, continuando con su función de promover niveles óptimos de calidad en los procesos de formación profesional, procedió a diseñar, implementar, validar y, finalmente, a publicar los Estándares y criterios de evaluación, con fines de acreditación de las carreras profesionales de los institutos y escuelas de educación superior tecnológicos y escuelas de formación técnico profesional de los sectores Defensa e Interior el 28 de noviembre de 2010. Antes de ello, ya se habían publicado los correspondientes al sector educación (21 de enero de 2009) y salud (26 de junio de 2010). En el caso de los IEST la norma señaló que la evaluación con fines de acreditación era voluntaria; sin embargo, la participación en estos procesos les ofrecía el reconocimiento formal de la calidad demostrada por cada una de las carreras profesionales de los mencionados institutos. El tipo de acreditación que correspondería a los IEST era acreditación por carrera profesional.

La elaboración de los Estándares y criterios de evaluación para la acreditación de las Carreras Profesionales de los IEST constituyó un espacio donde promotores, directores, docentes, especialistas, los funcionarios $y$ los académicos, considerados actores clave involucrados en la formación de profesionales en los IEST participaron activamente tanto del proceso de elaboración como de validación. Esta experiencia permitió sensibilizar, lograr consensos y trabajar por un objetivo común: lograr la calidad en los IEST. "Contribuyó a establecer consensos sobre la calidad educativa de las carreras profesionales y hacer objetivas y concretas las necesidades de mejora." (CONEACES, 2010a, p. 3)

El CONEACES procedió a la formulación del Proyecto de Estándares y Criterios de Evaluación para la Acreditación de las Carreras Profesionales de los Institutos y Escuelas de Educación Superior Tecnológicos.

En setiembre de 2009, se dio inició al proceso de validación, el cual tuvo tres etapas: (CONEACES, 2010b)

A. Primera etapa: estuvo orientada a acciones de sensibilización y validación con la participación de los representantes de los grupos de interés.

Se convocaron a reuniones de trabajo con expertos nacionales de los institutos y escuelas de educación superior tecnológico como: SENATI, CIBERTEC, TECSUP, CISE y Le Cordón Bleu Perú. Estas reuniones se llevaron a cabo en diciembre de 2009. Asimismo, se desarrollaron talleres macrorregionales de febrero a mayo de 2010 en Iquitos, Arequipa, Huancayo, Trujillo y Lima, según se observa en la Tabla 3. Los 450 participantes fueron elegidos considerando la dispersión de los IEST tanto públicos como privados.

Tabla 3

Número de IEST y personas que asistieron a los Talleres de validación de los estándares y criterios de evaluación para la acreditación de las carreras profesionales de los IEST

\begin{tabular}{llll}
\hline Sedes & Regiones & IEST & $\begin{array}{l}\text { Personas } \\
\text { asistentes }\end{array}$ \\
\hline Lima & $\begin{array}{l}\text { Lima Metropolitana } \\
\text { Lima provincias }\end{array}$ & 73 & 83 \\
& & &
\end{tabular}




\begin{tabular}{|c|c|c|c|}
\hline Iquitos & $\begin{array}{l}\text { Loreto } \\
\text { San Martín } \\
\text { Ucayali }\end{array}$ & 71 & 73 \\
\hline Arequipa & $\begin{array}{l}\text { Arequipa } \\
\text { Cuzco } \\
\text { Moquegua } \\
\text { Tacna } \\
\text { Madre de Dios } \\
\text { Apurímac } \\
\text { Puno }\end{array}$ & 162 & 162 \\
\hline Huancayo & $\begin{array}{l}\text { Ayacucho } \\
\text { Cerro de Pasco } \\
\text { Huancavelica } \\
\text { Huánuco } \\
\text { Junín }\end{array}$ & 76 & 76 \\
\hline Trujillo & $\begin{array}{l}\text { Amazonas } \\
\text { Ancash } \\
\text { Cajamarca } \\
\text { La Libertad } \\
\text { Lambayeque } \\
\text { Piura } \\
\text { Tumbes }\end{array}$ & 55 & 56 \\
\hline TOTAL & & 437 & 450 \\
\hline
\end{tabular}

B. Segunda etapa: tuvo como propósito recibir aportes relacionados con el aseguramiento de la calidad educativa. Para ello, se convocó a expertos nacionales (representantes de instituciones $y$ personalidades de reconocida trayectoria) quienes con objetividad y criterio ético brindaron sugerencias orientadas a mejorar la propuesta. Un ejemplo de ello fue la reunión realizada el 27 de octubre de 2010, donde se contó con la participación del Viceministro de Gestión Pedagógica, Dr. Idel Vexler Talledo; el Director de la DIGESUTP, Mg. Manuel Solís Gómez; el representante del Directorio del CONEACES, Ing. Guillermo Salas Donohue y los representantes de las organizaciones acreditadoras $y$ certificadoras en Sistemas de Calidad, Bureau Veritas, Estratega Consulting, Germanischer Llyd, Qualitas del Perú, SGS, AVANTÍA, INCOTEC, CERPER, Pricewaterhouse Coopers, Instituto para la Calidad-PUCP, TUV RHEILAND, CDI - Sociedad Nacional de Industrias, IPSOS APOYO Consultoría e International
Zeta Consulting, entre otras. Todas ellas interesadas en contribuir en la mejora de la calidad.

A nivel internacional, se contó con la presencia del doctor José María Ruiz Ruiz experto en análisis de asuntos relacionados con la calidad educativa, procedente de la universidad Complutense de Madrid y miembro de Team Europa. Él manifestó que el modelo era adecuado: "Estos modelos de última generación enfatizan las dimensiones relacionadas con los resultados e impactos del producto educativo en la sociedad" (CONEACES, 2010 b, p. 8). Asimismo, indicó que las actividades del CONEACES debían ser flexibles y firmes debido a las asimetrías que existían entre los IEST públicos y privados, urbanos y rurales, locales $y$ regionales. Finalmente, señaló que el Proyecto Educativo Institucional debía evidenciar con claridad la coherencia entre estrategia institucional y la asignación de recursos. Esta actividad se realizó en junio de 2010. 
C. Tercera etapa: tuvo como intención aplicar una experiencia piloto en dos institutos: Instituto de Educación Superior Tecnológico Público "Cueto Fernandini" y el Instituto de Educación Superior Tecnológico Privado CEADEX, uno de gestión publica y otro de gestión privada. Las experiencias permitieron determinar el nivel de operacionalización de los estándares en un ambiente y condiciones similares al de un instituto o escuela en proceso de autoevaluación.

Finalmente, se publicó el documento final: Estándares y criterios de evaluación aprobados para la acreditación de las carreras profesionales de los institutos y escuelas de educación superior tecnológicos y escuelas de formación técnico profesional de los sectores Defensa e Interior, el cual como parte de la metodología de CONEACES está sujeto a revisión periódica y actualización.

Con respecto al Modelo de Aseguramiento de la Calidad para la Acreditación de los IEST, este se planteó desde un enfoque de procesos como se observa en las Figuras 1 y 2 , el cual involucraba retroalimentación, con entradas y salidas claramente definidas para su innovación y mejoramiento. Se definió como holístico y multidimensional.
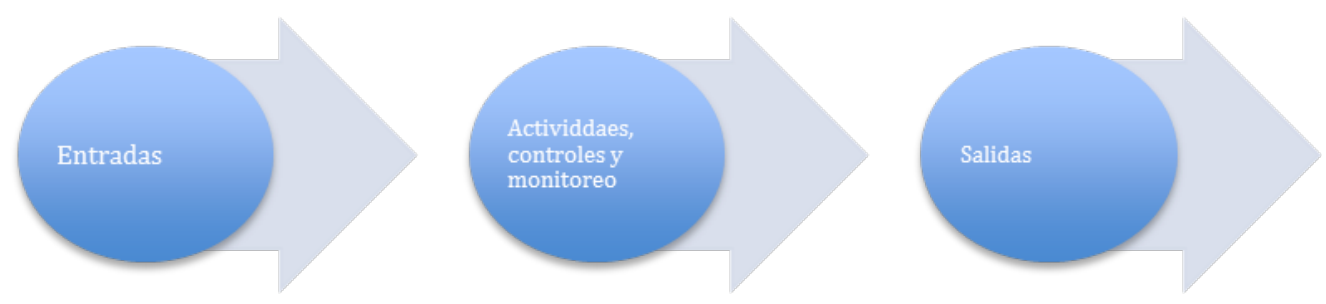

Figura 1. Enfoque de procesos del modelo de aseguramiento de la calidad
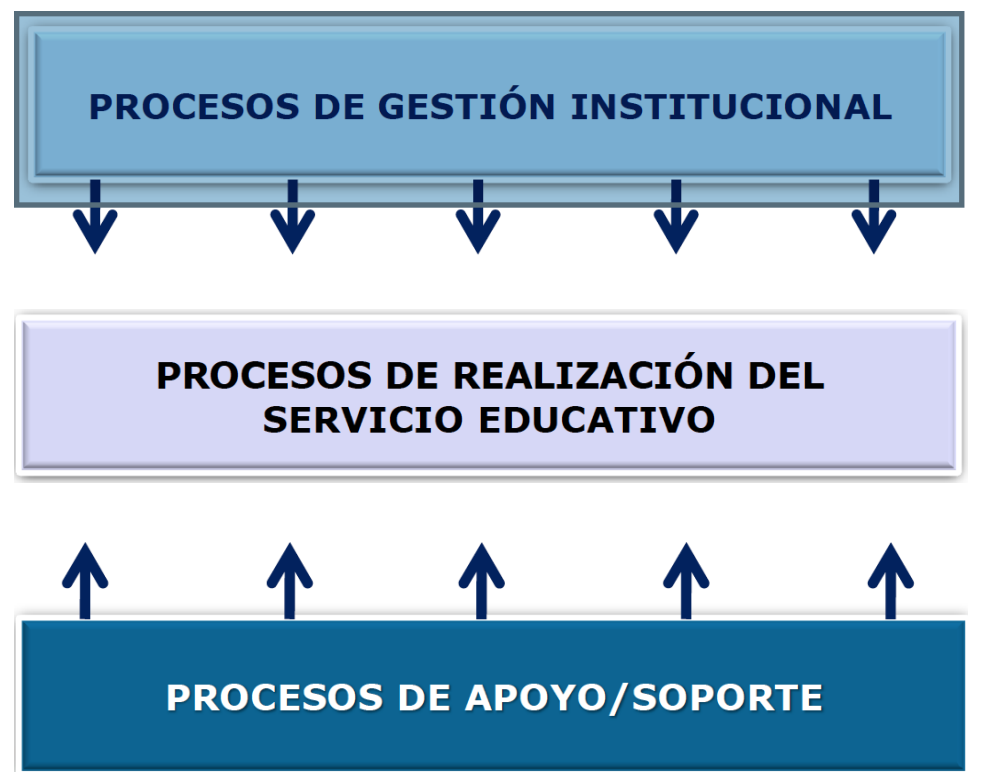

Figura 2. Enfoque de procesos del modelo de aseguramiento de la calidad

El propósito era movilizar procesos de cambio que partiesen de una evaluación interna, autoevaluación orientada a la autorregulación y mejora continua, y se dirigiesen a una externa, imparcial y objetiva de su cumplimiento. Por lo tanto, la evaluación obtuvo un carácter progresivo y sistemático, que calificaba la vigencia operativa y temporal, contrastada con los estándares y criterios de evaluación que establecía el CONEACES. 
El Modelo de Aseguramiento de la Calidad de CONEACES tiene cuatro dimensiones fundamentales (gestión institucional; procesos académicos; servicios de apoyo para la información profesional y resultados e impactos) que se descomponen en 17 factores y 71 estándares específicos orientados a medir el nivel de cumplimiento del estándar de calidad como se observa en la Figura 3.

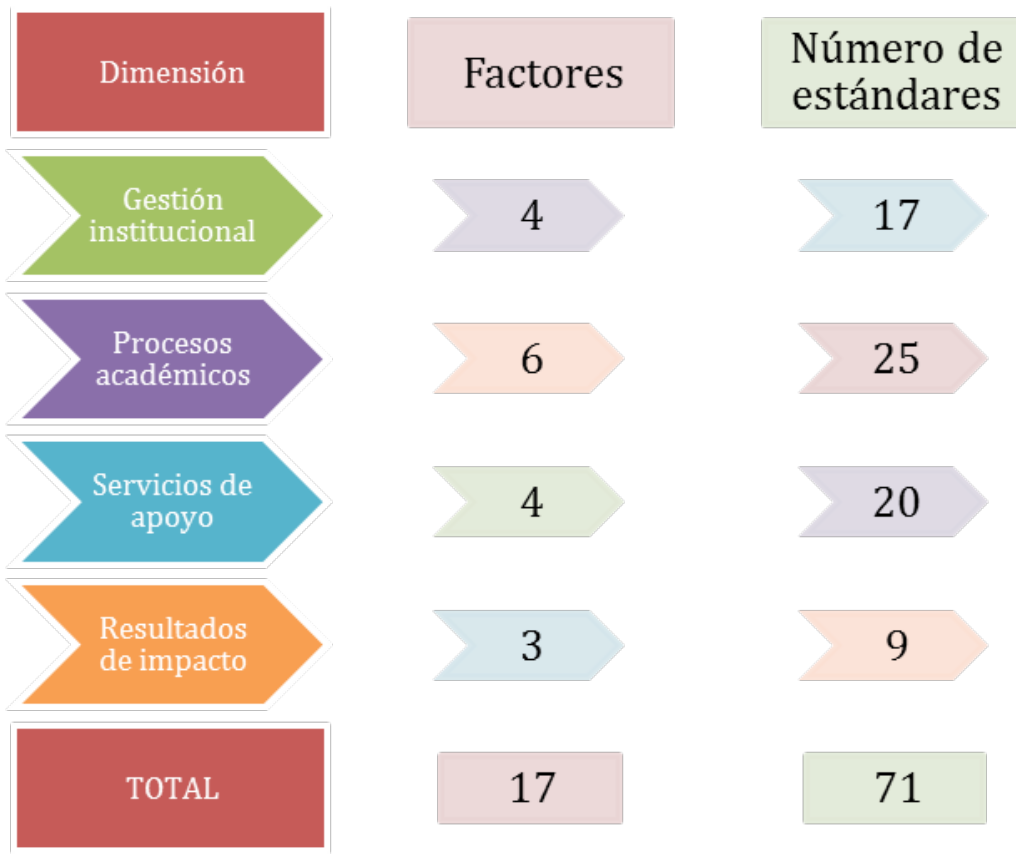

Figura 3. Dimensiones, factores y número de indicadores de los estándares de los IEST Fuente: CONEACES, 2012, p. 23

Al comparar las dimensiones de los IEST con las del modelo de los IFD, estas se diferencian por un estándar: IFD tiene 70 y IEST 71. Pero al comparar factores y estándares también se observan diferencias: en los IEST se denomina al estándar 1.3 .5 a formación y evaluación continua, mientras que en los IFD se le llama formación continua; a nivel de factores se denomina consejería, mientras que en los IFD se les llama tutoría. Finalmente, en los estándares del factor Infraestructura, equipamiento y tecnología se presentan 9 estándares en los IEST y 8 en los IFD. Esto se debe a que en los primeros se diferencian entre local y equipamiento, mientras que en el segundo solo se hace referencia al local. La diferencia se debe a la naturaleza de las carreras que forman ambas instituciones.

Los principios rectores de este proceso fueron la transparencia, la eficiencia, la eficacia, la pertinencia, la responsabilidad, la participación, la objetividad e imparcialidad, la ética y la periodicidad. (CONEACES, 2010b)

\subsection{Capacitación y monitoreo}

Una vez aprobados los estándares, CONEACES dio inicio a las capacitaciones de los docentes, especialistas y autoridades de los IEST sobre el proceso de evaluación y acreditación y todo lo que ello implicaba. La capacitación fue descentralizada. Las primeras capacitaciones constituyeron una estrategia de acercamiento a los IEST mediante jornadas intensivas de un día en la que se abordaron tres temas clave: (CONEACES, 2013a)

- Modelo de Calidad del CONEACES

- ¿Cómo evaluar los estándares de calidad? (Criterios a tener en cuenta para evaluar el nivel de calidad educativa)

- Procedimientos para la Acreditación 
La capacitación incluyó en una primera etapa 60 IEST en doce regiones del Perú (norte: Ancash, Cajamarca, Trujillo y Chiclayo; sur: Ayacucho, Cuzco, Tacna y Moquegua; centro:
Apurímac, Pasco, Junín y Huánuco, Lima). En la Tabla 4 se identifican los primeros IEST cuyos comités de calidad fueron capacitados.

Tabla 4

Primeros IEST, cuyos comités de calidad fueron capacitados.

\begin{tabular}{|l|l|}
\hline $\begin{array}{l}\text { Diciembre } \\
2010\end{array}$ & $\begin{array}{l}\text { Participación en la capacitación en el Modelo de Aseguramiento de la Calidad } \\
\text { al Instituto Superior Tecnológico KIPU del Cusco en el mes de diciembre. }\end{array}$ \\
\hline 14 - junio 2011 & $\begin{array}{l}\text { Evento de sensibilización al instituto de Educación Superior Tecnológico del } \\
\text { Ejército de Perú, el 14 de junio 2011. }\end{array}$ \\
\hline $\begin{array}{l}23 \text { y 24 junio } \\
2011\end{array}$ & $\begin{array}{l}\text { Capacitación de los Comités de Calidad del Instituto de Educación Superior } \\
\text { Tecnológico Eleazar Guzmán Barrón de Huaraz, el 23 y 24 de junio 2011. }\end{array}$ \\
\hline $\begin{array}{l}30 \text { - junio } \\
2011\end{array}$ & $\begin{array}{l}\text { Evento de sensibilización al Instituto de Educación Superior Tecnológico de la } \\
\text { Policía Nacional del Perú, el 30 de junio 2011. }\end{array}$ \\
\hline 8 - julio 2011 & $\begin{array}{l}\text { Evento de sensibilización al Instituto de Educación Superior Tecnológico } \\
\text { Daniel Alcides Carrión, el 08 de julio 2011. }\end{array}$ \\
\hline
\end{tabular}

CONEACES (2011, p. 21)

Fuente: CONEACES (2011). Reporte de gestión: Febrero - 2008 julio - 2011

A finales del año 2011, 9 IEST habían iniciado su proceso de autoevaluación. CONEACES en ningún momento ha dejado de brindar apoyo a las IEST ni de mantener el diálogo con sus comités de calidad. Ese interés de CONACES se debe a que entiende la evaluación como un proceso de mejora continua, donde todos quienes participan desde sus diversos roles deben mantenerse interactuando para retroinformarse y continuar elevando los niveles de calidad. Con esta mira realiza una actividad de apoyo continuo:
En estos cinco años, para promover la acreditación se han visitado 15 Regiones y se han llevado a cabo más de 60 Talleres, logrando la participación de cerca de 1,000 Institutos y Escuelas de Educación Superior de todo el País, con más de 2,000 participantes entre promotores, directores y docentes, obteniendo como resultado que más de 150 Instituciones han conformado sus Comités de Calidad e iniciado la etapa de Autoevaluación. (CONEACES, 2013b, 4)

Tabla 5

Capacitaciones dirigidas a los comités de calidad de los IEST en 2013

\begin{tabular}{|c|c|c|c|c|c|c|c|c|}
\hline \multirow[t]{2}{*}{ Trimestre } & \multirow{2}{*}{$\begin{array}{c}\text { Total de } \\
\text { actividades }\end{array}$} & \multirow{2}{*}{$\begin{array}{c}\text { Total de } \\
\text { Horas }\end{array}$} & \multicolumn{2}{|c|}{ Total de Institutos } & \multicolumn{3}{|c|}{ Total por tipo de IES } & \multirow{2}{*}{$\begin{array}{c}\text { Total de } \\
\text { participantes }\end{array}$} \\
\hline & & & Pub. & Priv. & Ped. & Salud & Otras & \\
\hline I & 03 & 18 & 02 & 01 & 01 & 01 & 01 & 90 \\
\hline II & 05 & 35 & 29 & 02 & 26 & 01 & 04 & 235 \\
\hline III & 19 & 144 & 73 & 36 & 41 & 08 & 60 & 1010 \\
\hline IV & 33 & 257 & 304 & 102 & 117 & 25 & 264 & 1968 \\
\hline TOTAL & 60 & 454 & 408 & 141 & 185 & 35 & 329 & 3303 \\
\hline
\end{tabular}

En 2013 se observó una actividad más intensa en el desarrollo de capacitaciones dirigidas a los comités de calidad. En todo el año se realizaron un total de 60 capacitaciones que ascendieron a un total de 454 horas como se puede observar en la Tabla 5. Dentro de este 
conjunto de capacitaciones 3303 participantes fueron beneficiados, los cuales pertenecían a 329 IEST. Se observó que en el cuarto trimestre se ofreció el mayor número de capacitaciones, lo que permitió inferir que el interés y el deseo de los IEST por lograr ser reconocidos como instituciones de calidad era latente.

El monitoreo se hizo presente mediante la ayuda técnica que siempre estuvo a disposición para brindar apoyo a los comités de calidad. El interés de los IEST hizo que rápidamente las autoridades viabilizaran estrategias para atenderlos, más aún, si la decisión de acreditarse era voluntaria. Para el efecto, durante 2013 se desarrollaron un número significativo de reuniones de ayuda técnica (62) como se observa en la Tabla 6.

Tabla 6

Reuniones de asistencia técnica ofrecida a los comités de calidad de los IEST durante 2013

\begin{tabular}{|c|c|c|c|c|c|c|c|c|}
\hline \multirow[t]{2}{*}{ Trimestre } & \multirow{2}{*}{$\begin{array}{c}\text { Total de } \\
\text { actividades }\end{array}$} & \multirow{2}{*}{$\begin{array}{c}\text { Total de } \\
\text { Horas }\end{array}$} & \multicolumn{2}{|c|}{ Total de Institutos } & \multicolumn{3}{|c|}{ Total por tipo de IES } & \multirow{2}{*}{$\begin{array}{c}\text { Total de } \\
\text { participantes }\end{array}$} \\
\hline & & & Pub. & Priv. & Ped. & Salud & Otras & \\
\hline II & 03 & 15 & & 03 & & & 03 & 30 \\
\hline III & 11 & 56 & 06 & 05 & 02 & 02 & 07 & 169 \\
\hline IV & 69 & 552 & 43 & 05 & 19 & 03 & 26 & 750 \\
\hline \multirow[t]{2}{*}{ TOTAL } & 83 & 623 & 49 & 13 & 21 & 05 & 36 & 949 \\
\hline & & & 62 & & 62 & & & \\
\hline
\end{tabular}

Las autoridades y equipos técnicos de CONEACES siempre se han mantenido cercanos a los IEST y a sus respectivos comités de calidad, muestra de ello ha sido el número de capacitaciones y ayudas técnicas ofrecidas. El éxito de estos procesos está en el acompañamiento que se hizo a las instituciones por vías presencial y, como ya se dijo, virtual.

\section{PROCESO DE EVALUACIÓN Y ACREDITACIÓN}

Los procesos de evaluación conducentes a la acreditación se desarrollan y concretan por carrera profesional y tiene carácter voluntario. Para dar inicio a estos procesos se debe cumplir con los siguientes requisitos: (SINEACE, 2014)

a. Informar sobre el inicio del proceso de autoevaluación con fines de acreditación, por cada carrera profesional de los IEST.

b. Designar al Comité de Calidad responsable del proceso de autoevaluación con fines de acreditación. En el caso de los IEST tendrán un Comité de Calidad por cada carrera profesional. Su conformación debe incluir a representantes de los grupos de interés y se sugiere que esté conformado por dos directivos, dos formadores, un administrativo, un egresado, un estudiante. Esta designación de la conformación del Comité de Calidad es comunicada al CONEACES.

c. Capacitar a los miembros del Comité de Calidad en la metodología de autoevaluación propuesta por el CONEACES.

Una vez cumplidos estos tres procedimientos la institución está en condiciones de dar inicio a sus procesos de evaluación.

\subsection{Autoevaluación}

La autoevaluación está liderada por el Comité de Calidad del programa o carrera profesional, con la participación de los grupos de interés. La autoevaluación se realiza considerando los niveles de cumplimiento de los estándares y criterios de evaluación aprobados y publicados por el CONEACES. 
Según SINEACE (2014a), respecto de los IEST, hay 537 carreras o especialidades que están en proceso de autoevaluación, de ellos el $70 \%$ son públicas y $30 \%$ son privadas. Las Escuelas Superiores de Formación Artística (ESFA) que se encuentran en ese proceso son 17.
En 2014 el número de IEST que han iniciado el proceso acreditación o se han acreditado asciende a 282 instituciones. En la Figura 4 se percibe que este proceso ha sido iniciado en todas las regiones del Perú.

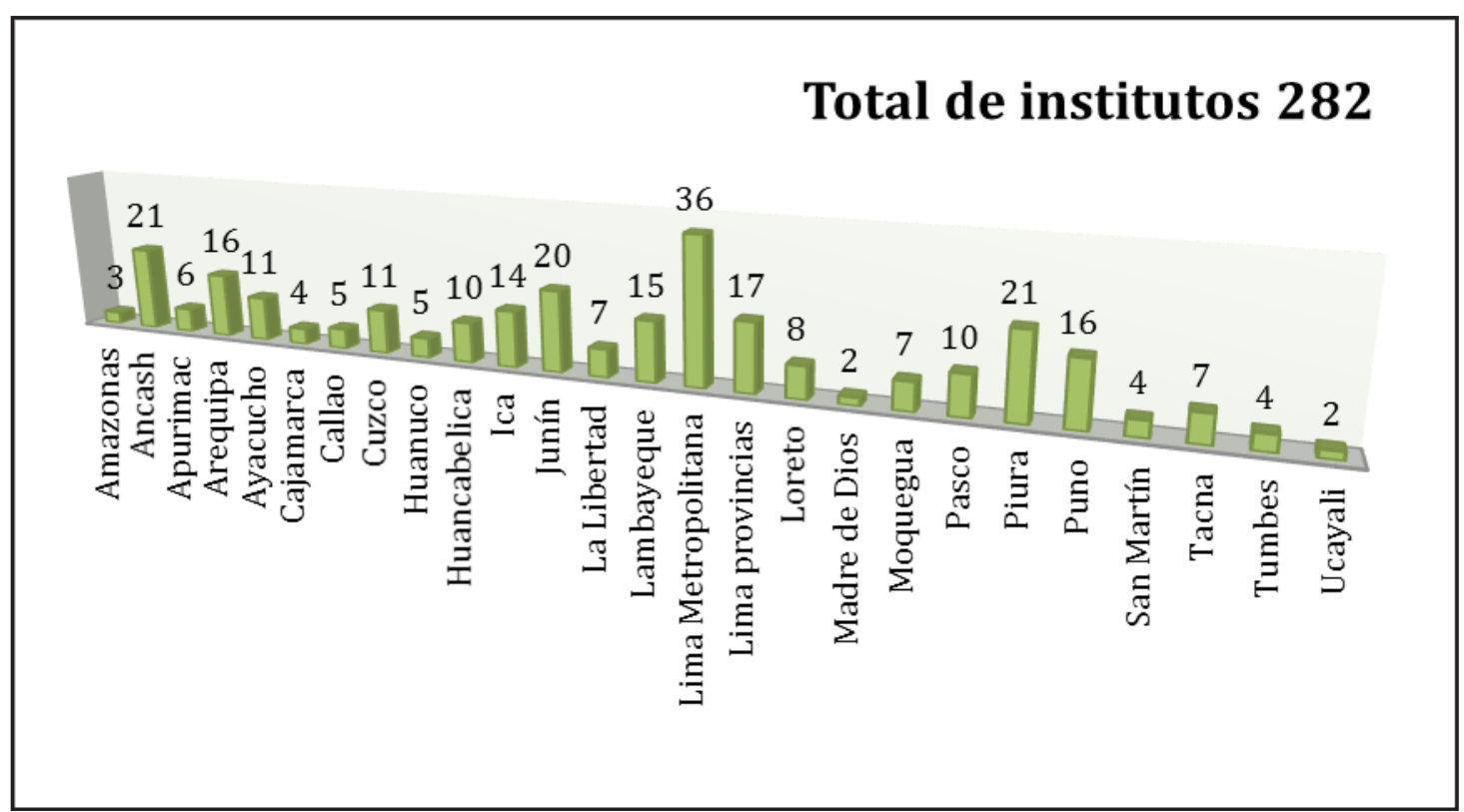

Figura 4: Total de institutos superiores tecnológicos acreditados y en proceso Fuente: SINEACE, 2014b, p. 11

En la Figura 5 se observa que 551 carreras ofrecidas por los IEST se encuentran en proceso de acreditación o ya fueron acreditados y que se encuentran distribuidos en todo el territorio nacional.

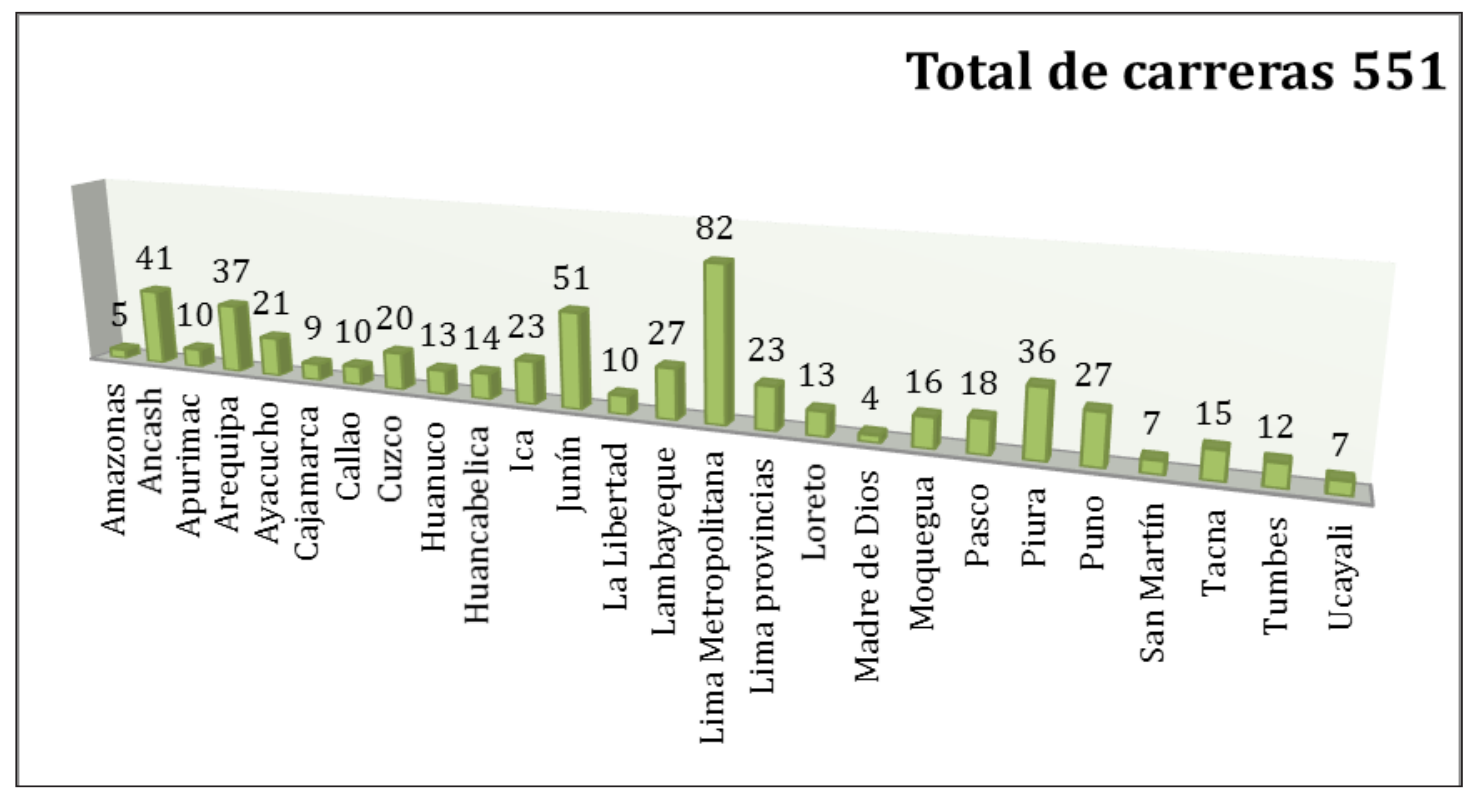

Figura 5: Total de carreras de institutos superiores tecnológicos acreditados y en proceso según regiones

Fuente: SINEACE, 2014b, p. 11 
Si bien los procesos de acreditaciones se han generalizado en todas las regiones, las acreditaciones se han concretado en Lima (82 carreras), Junín (51 carreras) y Ancash (41 carreras), como se observa en la Figura 5.
En la Figura 6 se muestra un avance significativo con respecto a los procesos de autoevaluación, evaluación externa y acreditación. Esto evidencia que los IEST en el Perú se encuentran en permanente movilización orientada a lograr la calidad del servicio que ofrecen.

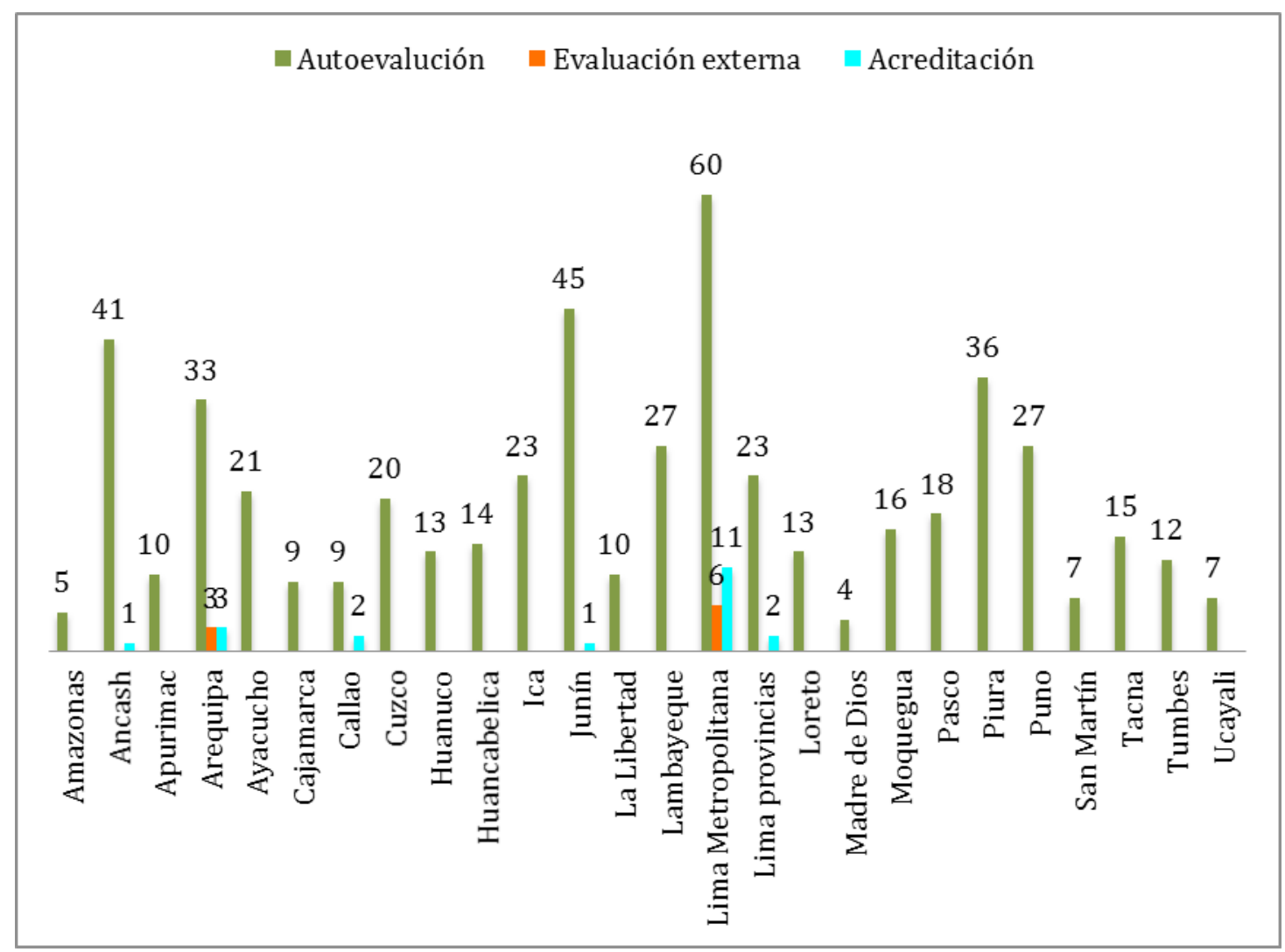

Figura 6. Número de IEST en autoevaluación, evaluación externa y acreditados Nota: SINEACE 2014b, p. 12

Al analizar, los resultados en la Figura 7, se evidencia que si bien el 4\% de IEST ya se acreditó, el $2 \%$ se encuentra en etapa de evaluación externa y el $94 \%$ está dedicado a su proceso de autoevaluación. Estos procesos se han convertido en una práctica generalizada y ya se instalaron en las instituciones.

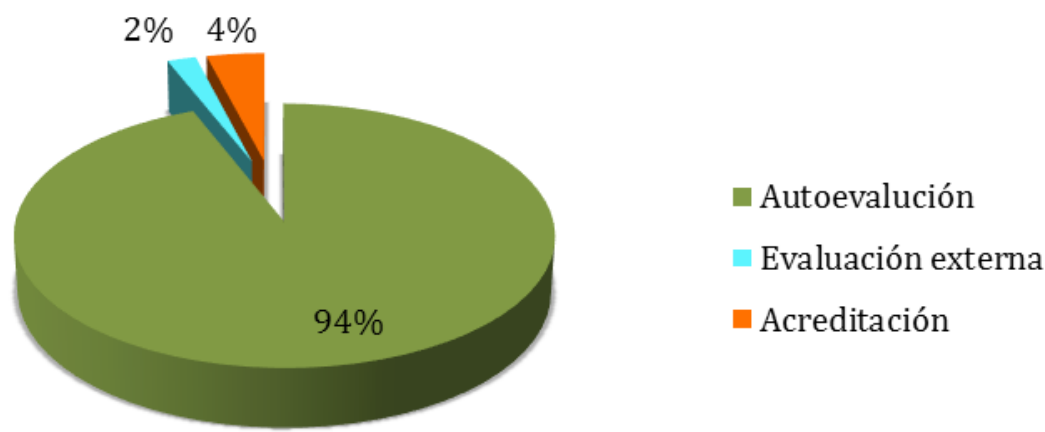

Figura 7. Número de IEST en autoevaluación, evaluación externa y acreditados Fuente: SINEACE 2014b, p. 12 
En la Figura 8 se aprecia que el 55\% (407) de IEST en proceso de acreditación son privados mientras que el $45 \%$ (335) públicos. Sin embargo, las instituciones que en mayor porcentaje han iniciado el proceso de acreditación o ya se acreditaron son las instituciones públicas con un $70 \%$ (196 IEST), mientras que las privadas solo un $30 \%$ (86).

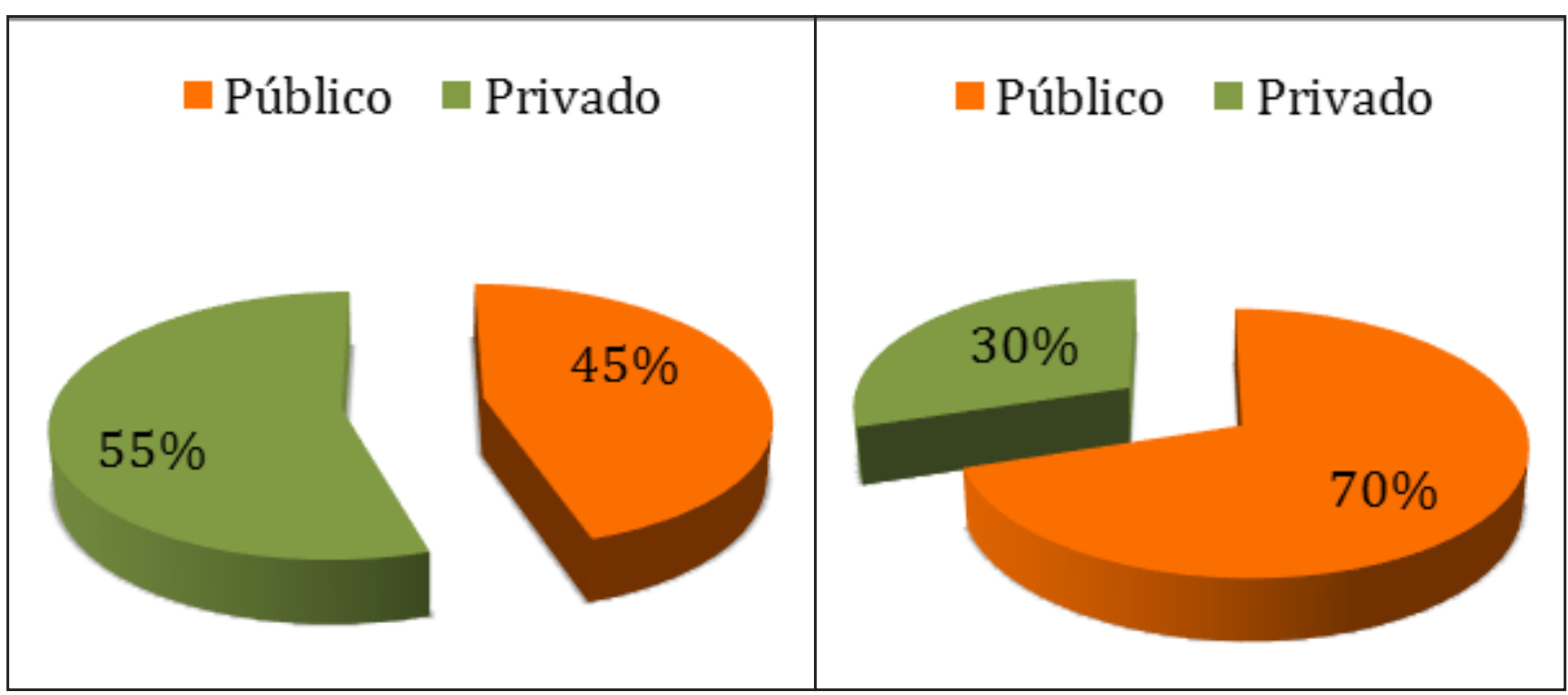

Figura 8. IEST en proceso de acreditación, por tipo de gestión Fuente: SINEACE, 2014b, p. 14

En la Figura 9 se observa que de los 282 IEST que iniciaron el proceso de acreditación, el 1\% de los IEST públicos alcanzó la acreditación, mientras que en los privados el $13 \%$ ha sido acreditado y el $6 \%$ se encuentra en la etapa de evaluación externa.

\section{Acreditación}

Ev. Externa

\section{Autoevaluación}

Públicos

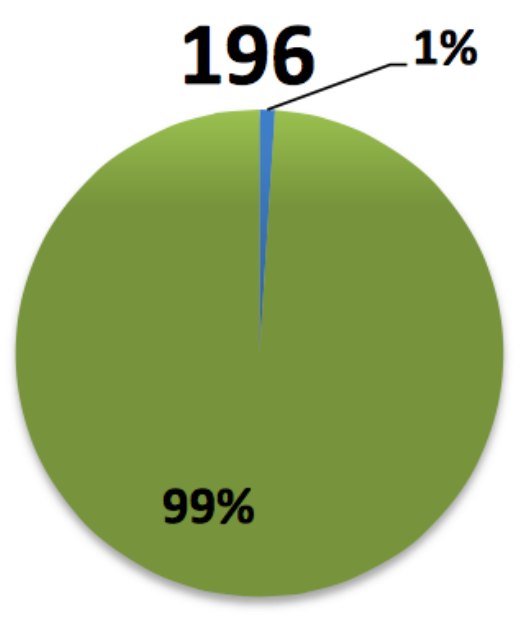

Privados

86

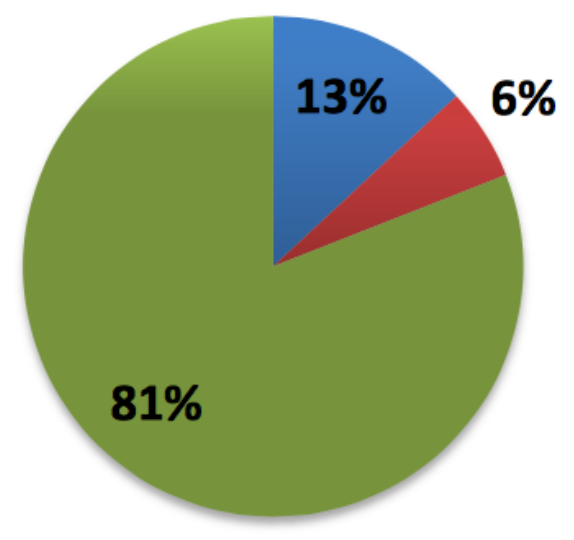

Figura 9. IEST en proceso de acreditación, por tipo de gestión Fuente: SINEACES, 2014b, p 15

Con respecto a las familias profesionales, se observa en la Figura 10 que luego de la familia de salud, se encuentras en avance de procedimientos de evaluación y acreditación las familias Administración y Comercio, Computación e Informática y Actividades Agrarias. 


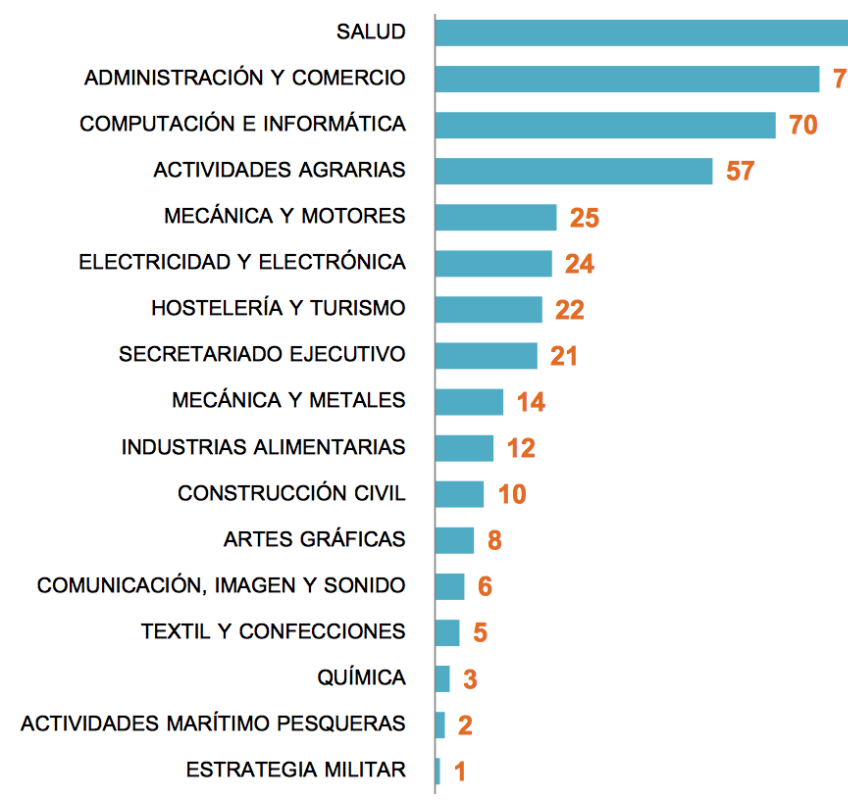

Figura 10. Total de carreras por familia profesional

Nota: SINEACE 2014b, p. 16

Las capacitaciones contaron con la colaboración del Proyecto ProCalidad a partir de 2014, el cual fue implementado por el Sistema Nacional de Evaluación, Acreditación y Certificación de la Calidad Educativa (SINEACE) e involucró a los órganos operadores del SINEACE: CONEAU y CONEACES. ProCalidad constituyó un Fondo de Mejoramiento de la Calidad que brindó estímulos económicos mediante un fondo competitivo para que las instituciones puedan contratar agencias de calidad para la evaluación externa $y$, además, puedan financiar sus planes de mejora. Este fondo contó con el apoyo del Banco Mundial.

Si bien se ha avanzado de manera significativa gracias al apoyo de las autoridades y el esfuerzo de los IEST, aun queda pendiente: lograr la acreditación de todas la instituciones que se encuentran en proceso de autoevaluación, asegurar los fondos para que este no sea un impedimento y motivar a la práctica continua de estos procesos.

\subsection{Evaluación externa: Instituciones aseguradoras}

El CONEACES continuó implementando los procesos conducentes a la acreditación. El 21 de mayo de 2010 publica la Guía de procedimientos para la autorización y registro de entidades evaluadoras con fines de acreditación. El 1 de setiembre de 2011 se propone la segunda versión y se oficializa el 6 de diciembre de 2012.

La evaluación externa es: "el proceso de verificación, análisis y valoración, que se realiza a un programa o a una institución educativa, a cargo de una entidad evaluadora, que cuenta con autorización vigente emitida por el CONEACES." (CONEACES, 2008, p. 27). Esta evaluación tiene como propósito constatar la veracidad de los resultados de la autoevaluación realizada por el comité de calidad de la carrera que desea acreditar el IEST.

El comité de calidad del IEST puede impugnar la composición de la comisión de evaluación, si se observase conflicto de interés con alguno de sus miembros, pues podría afectar imparcialidad del proceso.

La etapa de evaluación externa se inicia con la solicitud de la carrera o programa que haya culminado su autoevaluación. Las entidades evaluadoras externas, previa autorización, conforman un equipo evaluador, realizan la visita de verificación, evalúan el informe y proponen la acreditación. El proceso se grafica en la Figura 11. 


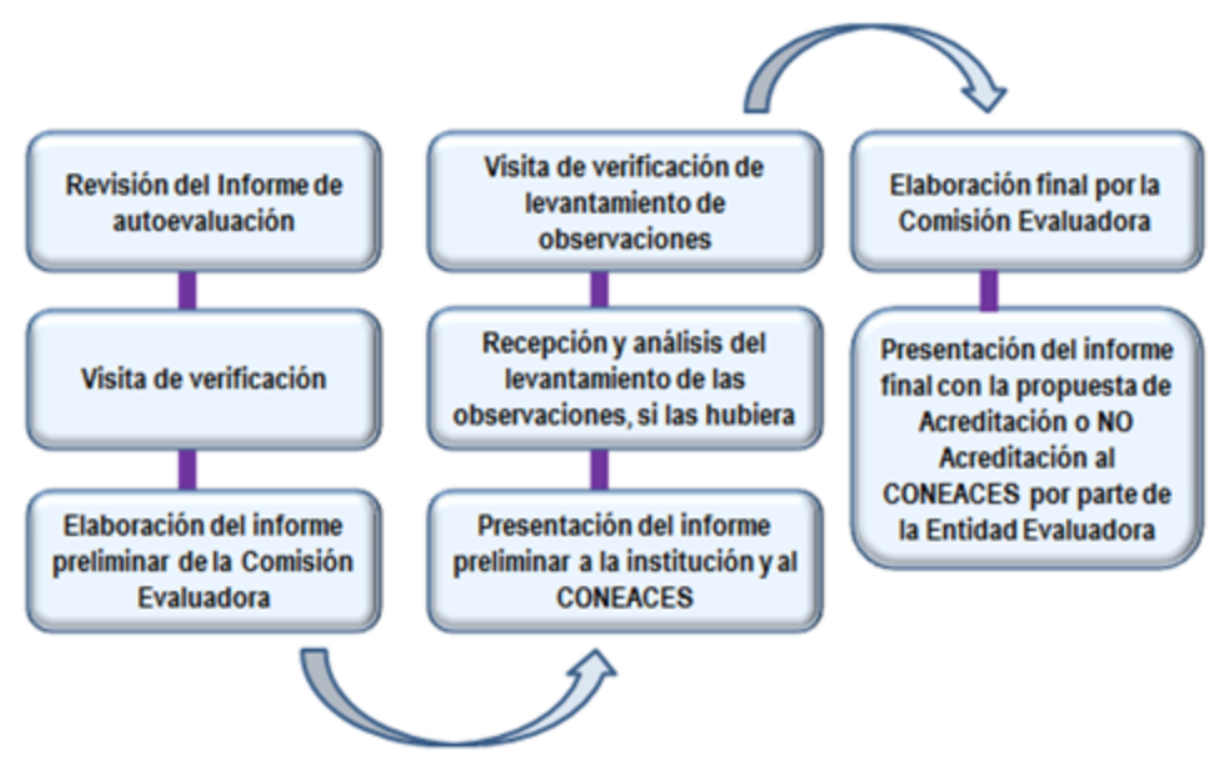

Figura 11. Procedimiento para la evaluación externa

Fuente: CONEACES, 2012, p. 43

El SINEACE ha autorizado a las siguientes Entidades Evaluadoras Externas para la verificar el cumplimiento de los estándares de aquellas carreras profesionales que soliciten ser evaluadas para obtener la acreditación.

- International Zeta Consulting

Resolución: № 002-2012-SINEACE/P

Vigencia: Noviembre 2011 a noviembre 2016

- SGS Certificadora de Educación SAC Resolución: $\mathrm{N}^{\circ}$ 008-2012-SINEACE/P Vigencia: Diciembre 2011 a diciembre 2016

- Beyond Quality Standards

Resolución:

$\mathrm{N}^{\circ}$ 037-2014-COSUSINEACE/CDAH-P

Vigencia: Diciembre 2014 a diciembre 2019.

- International Zeta Consulting fue la primera empresa acreditada por el CONEACES como entidad evaluadora con fines de acreditación.

El CONEACES tuvo acogida por las empresas privadas, las que no dudaron en implementar los procesos necesarios constituirse como entidades evaluadoras. Debe recordarse, que alguna de ellas participó en los procesos de validación de los estándares.

\subsection{Instituciones acreditadas}

Se publicaron los documentos normativos $y$ orientadores para conducir los procesos de evaluación orientados a la acreditación:

\section{A. Estándares}

- Estándares y criterios de evaluación para la acreditación de las carreras profesionales de los institutos y escuelas de educación superior tecnológicos y escuelas de formación técnico profesional de los sectores de Defensa e Interior

- Criterios, estándares, indicadores y proceso de evaluación para la revalidación de los institutos y escuelas de educación superior.

\section{B. Documentos técnicos}

- Procedimiento para la evaluación y acreditación de las instituciones de educación superior no universitaria. (Versión I y II) 
- Guía de autoevaluación para la acreditación de los institutos y escuelas de educación superior

- Procedimiento para la emisión de opinión de conveniencia de las carreras profesionales que propongan ofrecer los institutos y escuelas de educación superior

- Guía de procedimiento para la autorización y registro de entidades certificadoras

Asimismo, se desarrollaron los talleres de capacitación a los comités de calidad. Finalmente, en 2012 el CONEACES estuvo en condiciones de proceder a la acreditación.

El primer IESP acreditado fue el Instituto Continental con sede en Huancayo. Este Instituto dedicó un año a acreditar seis de ocho carreras: Administración. Administración Bancaria, Secretariado Ejecutivo, Computación, Diseño Gráfico y Gastronomía y Arte Culinario. La Directora del Instituto señaló:

Para nuestros egresados y nuestros estudiantes ser formados en un instituto que ha sido reconocido por el Estado peruano que está brindando servicios de calidad, les da ventajas de tener mayor recepción en las empresas, en las organizaciones. (RPP Noticias, 2013, p. 7)

Solo tres institutos superiores han certificado su calidad educativa.

La Asociación de Institutos Superiores Tecnológicos y Escuelas Superiores del Perú (ASISTEPERÚ) es una Asociación que congrega a 21 IEST privados del Perú, los cuales son considerados los más prestigiosos a nivel nacional y tienen el $35 \%$ de la matrícula del total del sector privado.

Esta Asociación contribuyó a que sus asociados participasen de los procesos de evaluación conducentes a la acreditación. En el primer trimestre de 2012, los 21 institutos afiliados a ASISTE PERÚ decidieron iniciar voluntariamente el proceso de autoevaluación, para acreditarse en el sistema local. Ellos consideraban que el resultado distingue a las instituciones que cumplen con los estándares. Luego de dos años de trabajo, los institutos siguientes institutos lograron acreditar sus carreras: Instituto de Formación Bancaria (IFB), Instituto Peruano de Administración de Empresas (IPAE), Instituto de Educación Superior Tecnológico Privado Toulouse Lautrec, Le Cordon Blue Perú, y el Centro de Estudios de Aviación Profesional (AVIA).

Hasta 2014 se han acreditado 45 carreras de un total de 18 IEST, se ha llegado a esta cifra de manera progresiva. En la Tabla 7 se muestra la relación de IEST acreditados.

Es importante destacar, que así como ASISTEPERÚ asumió el reto de la acreditación, también lo hicieron instituciones que pertenecían al sector Defensa. Una de ellas fue el Instituto de Educación Superior Tecnológico Naval - CITEN (CITEN). Este Instituto acreditó la carrera de Electrónica en febrero de 2014. El Instituto de Educación Superior Tecnológico Público del Ejército - ETE "Sgto. 2do Fernando Lores Tenazoa” (ETE) acreditó, en junio de 2014, tres carreras: Administración, Telecomunicaciones, Mecánica Automotriz con una puntuación de 305 puntos. Esta fue la tercera institución del sector Defensa en acreditarse. Ambas instituciones continúan preparándose para acreditar todas las carreras $y$ especialidades que se ofrecen en ellas.

Todos los IEST acreditados emiten anualmente un informe sobre los proceso de implementación de mejora. La acreditación tiene una duración de tres años.

$\mathrm{Si}$ bien las autoridades de SINEACE viabilizaron el apoyo técnico a los IEST, eran conscientes de que no era suficiente, también se necesitaba apoyo económico. En agosto de 2014 el SINEACE a través de su Proyecto "Mejoramiento de la Calidad de la Educación Superior" (ProCalidad) lanzó el primer concurso de financiamiento para Institutos y Escuelas de Educación Superior con el propósito de impulsar los procesos de acreditación de la calidad educativa. La subvención estuvo dirigida hacia el 
financiamiento de los planes de mejora de carreras con fines de acreditación, así como para evaluaciones externas con fines de acreditación de carreras con un monto de hasta S/. 350000 soles. Además, las carreras que ya hubieran logrado su acreditación, pero que estuviesen en búsqueda de mayores logros este fondo, podía financiar hasta S/. 690000 soles.
Los ganadores del Primer Concurso a nivel IEST, en la primera modalidad fue el IEST Ejército ETE Sggto. 2do Fernando Lores Tenazoa por las carreras de Mecánica Automotriz y Enfermería Técnica. Esta carreras recibieron S/. 134162 y S/. 346500 respectivamente. En la segunda modalidad, resultaron ganadores el ISE Pomacanchi del Cusco, recibió un fondo $\mathrm{de}$ S/.700 000 soles.

Tabla 7

IEST y las carreras tecnológicas acreditadas hasta 2014

\begin{tabular}{|c|c|c|}
\hline \multicolumn{3}{|c|}{ CARRERAS TECNOLÓGICAS ACREDITADAS } \\
\hline \multirow[t]{6}{*}{01} & Instituto Continental & 01. Computación e Informática \\
\hline & (Huancayo) & 02. Diseño Gráfico \\
\hline & & 03. Secretariado Ejecutivo \\
\hline & & 04. Administración Bancaria \\
\hline & & 05. Administración \\
\hline & & 06. Gastronomía y Arte Culinario \\
\hline \multirow[t]{3}{*}{02} & Instituto de Formación & 07. Administración Bancaria (Sede Norte) \\
\hline & Bancaria (IFB) & 08. Administración Bancaria (Sede San Juan de Lurigancho) \\
\hline & & 09. Administración Bancaria (Sede Principal)(vigencia: 3 años) \\
\hline 03 & $\begin{array}{l}\text { ISTP María Montessori } \\
\text { (Arequipa) }\end{array}$ & 10. Educación Inicial y Primaria \\
\hline 04 & CITEN (Callao) & 11. Electrónica (vigencia: 3 años) \\
\hline 05 & AVIA & 12. Administración de Servicios de Hostelería (vigencia: 3 años) \\
\hline \multirow[t]{2}{*}{06} & Toulouse Lautrec & 13. Dirección y Diseño Gráfico (vigencia: 3 años) \\
\hline & & 14. Arquitectura de Interiores (vigencia: 3 años) \\
\hline 07 & IPAE & $\begin{array}{l}\text { 15. Administración de Negocios (Sede Pueblo Libre) (vigencia: } 3 \text { años). } \\
\text { 16. Administración de Neoocios (Sede Surco) (videncia } 3 \text { años) }\end{array}$ \\
\hline \multirow[t]{3}{*}{08} & Le Cordon Bleu Perú & 17. Administración de Hoteles, Restaurantes y Afines (vigencia: 3 años). \\
\hline & & 18. Cocina (vigencia: 3 años). \\
\hline & & 19. Gastronomía y Arte Culinario (vigencia: 3 años). \\
\hline 09 & SISE & 20. Administración de Negocios (vigencia: 3 años). \\
\hline \multirow[t]{6}{*}{10} & Instituto de Educación & 21. Administración (vigencia: 3 años). \\
\hline & Superior Tecnológico & 22. Telecomunicaciones (vigencia: 3 años). \\
\hline & Público del Ejército - & 23. Mecánica automotriz (vigencia: 3 años). \\
\hline & ETE "Sgto. 2do & \\
\hline & Fernando Lores & \\
\hline & Tenazoa" & \\
\hline 11 & $\begin{array}{l}\text { Instituto San Ignacio de } \\
\text { Loyola }\end{array}$ & 24. Administración de Hoteles y Restaurantes (vigencia: 3 años) \\
\hline & $\begin{array}{l}\text { Instituto del Sur } \\
\text { (Arequipa) }\end{array}$ & $\begin{array}{l}\text { 25. Administración Bancaria y Financiera (vigencia: } 3 \text { años). } \\
\text { 26. Gastronomía y Arte Culinario (vigencia: } 3 \text { años). }\end{array}$ \\
\hline & Instituto CIBERTEC & 27. Administración de Empresas (vigencia: 3 años). \\
\hline & Sede Norte & 28. Diseño Gráfico (vigencia: 3 años). \\
\hline & & 29. Redes y Comunicaciones (vigencia: 3 años). \\
\hline & & 30. Computación e Informática (vigencia: 3 años). \\
\hline \multirow[t]{8}{*}{14} & Instituto de & 31. Computación e Informática \\
\hline & Educación Superior & 32. Gastronomía y Arte Culinario \\
\hline & Público Chincha & 33. Administración de Empresas \\
\hline & & 34. Guía Oficial de Turismo \\
\hline & & 35. Contabilidad \\
\hline & & 36. Educación Inicial \\
\hline & & 37. Educación Secundaria: Idioma Inglés \\
\hline & & 38. Educación Secundaria: Educación Física \\
\hline
\end{tabular}




\begin{tabular}{lll}
\hline 15 & $\begin{array}{l}\text { Instituto de Educación } \\
\text { Superior Tecnológico } \\
\begin{array}{l}\text { Público Huando } \\
\text { (Huaral) }\end{array}\end{array}$ \\
\hline 16 & $\begin{array}{l}\text { Instituto De Formación } \\
\text { Bancaria (IFB) - Sede }\end{array}$ & 40. Administración de Negocios Internacionales \\
\hline & Norte & \\
\hline 17 & Instituto Chío Lecca & 41. Diseño de Modas \\
\hline 18 & Instituto Superior de & 42. Computación e Informática \\
& Educación Público La & 43. Secretariado Ejecutivo \\
& Inmaculada (Camaná) & 44. Educación Inicial \\
& 45. Educación Primaria \\
\hline
\end{tabular}

Nota: No se han considerado las carreras de Salud

Además de ellos, también calificaron el IEST Naval CITEN por su carrera de Telemática, al IEST Otuzco de la región La Libertad por su carrera de Enfermería Técnica y al IEST Ejército ETE Sgto. 2do Fernando Lores Tenazoa por la carrera de Mecánica de Producción. En este caso, el primero recibió S/. 321 862; el segundo, S/. 346 500, y el ultimo, S/. 346420.

La segunda convocatoria estuvo dirigida a universidades. En enero de 2015 se anunció la tercera convocatoria y considera las mismas modalidades y se les ha dado el nombre de: "Iniciación al cambio", dirigido a aquellos IEST que no han sido acreditados, pero que cuentan con un informe de autoevaluación o de evaluación externa; y "Camino a la excelencia", para los que ya cuentan con su respectiva acreditación, pero desean encaminarse hacia la excelencia.

Definitivamente, la búsqueda de la calidad se ha convertido en un imperativo para los IEST, las autoridades del sector educación y de la sociedad en general. Se puede afirmar que la calidad es parte de la cultura de las instituciones acreditadas $y$ se evidencia en las diferentes dimensiones que incluye el proceso formativo: gestión, proceso académicos, servicios de apoyo y resultados de impacto. Los profesionales egresados de estas instituciones estarán en condiciones de satisfacer las demandas del mercado laboral, de competir y contribuir en el desarrollo del Perú y su proceso de transformación.

4. Reflexión final

Los procesos de transformación experimentados en el Perú en las últimas décadas han generado nuevas demandas para poder continuar con el desarrollo de forma sostenida, y para ello es una prioridad ofrecer una formación de calidad. Al respecto Luce y Morosini (2004) afirman:

La formación de ciudadanos $y$ profesionales que sean capaces de construir una sociedad más justa $e$ igualitaria; es el compromiso de la sociedad y de sus representantes a partir de la inversión publica y privada en el conocimiento, la ciencia y la tecnología. (Fernández, s.f., p. 35)

El SINEACE se creó con el propósito de implementar sistemas de evaluación y acreditación que fueran coherentes con sus objetivos de regulación y generasen una cultura de la evaluación como estrategia para la mejora continua de la calidad educativa. Para ello fue necesario articular de manera progresiva el marco legal que le ha dado sustento, esto incluyó desde la Constitución Política del Perú, la Ley General de Educación, entre otras normas, hasta la creación del Sistema Nacional de Evaluación, Acreditación y Certificación de la Calidad Educativa: Ley 28740 en 2006 y su Reglamento en 2007, donde se nomina a los órganos operadores.

Para atender específicamente a los IEST se implementó el Consejo de Evaluación, Acreditación y Certificación de la Calidad de la Educación Superior No Universitaria autorizado por Decreto Supremo N. ${ }^{\circ}$ 0182007- ED. El CONEACES, desde un inicio, asumió el compromiso de crear en los IEST una cultura de calidad a partir de la mejora continua. No escatimó esfuerzos para convocar 
a las cerca de 1000 instituciones para que hicieran suya esta intención.

El trabajo realizado por CONEACES se sustentó en los lineamientos que articularon lo teórico- conceptual y lo metodológicooperativo, los cuales permitieron diseñar el modelo de aseguramiento de la calidad que respondiera a la heterogeneidad que caracterizaba a los IEST. Una vez implementado el modelo se procedió a su operacionalización mediante la construcción de los estándares y criterios de evaluación para la acreditación de las carreras profesionales de los IEST.

El CONEACES optó como estrategia el trabajo participativo y tuvo la capacidad de convocatoria necesaria para involucrar en la implementación de los procesos de evaluación y acreditación a todos los actores relacionados con los IEST: autoridades, promotores, directores, docentes, especialistas, funcionarios, académicos, expertos nacionales $e$ internacionales, es decir la comunidad educativa en pleno. Esta convocatoria fue la fortaleza que contribuyó a sensibilizar con respecto a la necesidad de trabajar con calidad. Evidencia de ello han sido los más de 200 IEST que hasta finales de 2014 se encontraban en proceso de autoevaluación, evaluación externa o se habían acreditado. Lo más seguro es que 2015 sea un año de muchas satisfacciones para CONEACES al acreditar a todas ellas.

Otro elemento que ha contribuido en el nivel de respuesta de los IEST, a pesar de que no estaban obligados a participar de estos procesos, es la disposición a brindarles capacitación y apoyo técnico de forma permanente. Se emplearon todos los canales disponibles presenciales (talleres, reuniones, foros, encuentros, etc.) como no presenciales (periódicos, teléfono, e-mail, página web, blog) Todo canal fue bueno, siempre y cuando se pudiera responder a las inquietudes de los comités de calidad. Es así como se han realizado más de 60 talleres de capacitación a nivel nacional.

El resultado de todo los esfuerzos realizados por CONEACES y los IEST por lograr la acreditación se ve reflejado en la satisfacción de las instituciones que se han acreditado y en los propios miembros del Órgano Operador.

Narda Ybañez, directora del Instituto Continental de Huancayo, manifestó: "Ser el primer Instituto tecnológico en el Perú que acredita 6 carreras, no es producto de la casualidad... es el resultado de más de dos décadas de trabajo... que se releja en nuestros egresados" (2013 , p. 2)

Karen Stiegler Directora de Innovación y Desarrollo de Toulouse Lautrec afirmó:

Los evaluadores de SGS entrevistaron a nuestros docentes, autoridades, alumnos y administrativos y comprobaron nuestra moderna gestión institucional, auditaron nuestros procesos académicos, verificaron los servicios de apoyo a la enseñanza y se sorprendieron con los sobresalientes resultados de impacto en la sociedad que tiene la carrera de Dirección y Diseño Gráfico. (2014, p. 5)

El Comandante General del Ejército del Perú, General de División Ronald Emilio Hurtado Jiménez, Director afirmó, luego de acreditar cuatro carreras del Instituto de Educación Superior Tecnológico Público del Ejército "Sgto. 2do Fernando Lores Tenazoa".

Todos queremos lograr la calidad educativa y esa calidad se logra siguiendo los procedimientos que nos van a permitir ser más eficientes. Por eso tenemos que ir formalizando todas nuestras carreras y gracias al sistema del SINEACE vamos a seguir avanzando. (Jiménez, 2014, p. 3)

Agregó:

Las acreditaciones ya logradas como un hecho "trascendente e histórico", ahora que el IESTPE - ETE cumple cuarenta años de funcionamiento, $\mathrm{y}$ [es] como el punto de partida de lo que esperan lograr en los años venideros en todas sus instituciones educativas. (Jiménez, 2014, p. 4) 
La presidenta del SINEACE, Peregrina Morgan Lora indicó: "Los estudiantes de estos institutos que se acreditan tienen la responsabilidad de ser de los mejores en el mundo laboral, tras haber adquirido competencias técnicas de primer nivel." (2014, p. 2)

Han sido seis años de trabajo ininterrumpido, cuyo producto se evidencia al contar con el sustento legal pertinente, estándares y criterios de evaluación y acreditación validados, materiales, capacitaciones, apoyo técnico, agencias de acreditación, comités de autoevaluación, IEST acreditados y satisfechos de la tarea realizada, autoridades comprometidas. En conclusión, CONEACES concilió con todos los actores de la educación superior no universitaria para trabajar juntos para asegurar una educación de calidad para los jóvenes peruanos. 


\section{REFERENCIAS}

Ángeles, N., Bacigalupo, J., Bardález, E., Campana, Y., Cuba, E., Diez, A., et al. (2012) La investigación económica y social en el Perú: Balance 2007-2011 y agenda 2012 - 2016. Lima, Perú: Consorcio de Investigación Económica y Social.

Consejo de Evaluación, Acreditación y Certificación de la Calidad de la Educación Superior No Universitaria. (2010a, 19 de julio) Estándares y criterios de evaluación para la acreditación de las carreras de salud de las instituciones y escuelas de educación superiores tecnológicas. El Peruano, pp. 1-12. En http://www.gacetajuridica.com.pe/servicios/normaspdf_2010/Junio/19-06-2010_ SE_EstAndares.pdf.

Consejo de Evaluación, Acreditación y Certificación de la Calidad de la Educación Superior No Universitaria (2008b) Estándares y criterios de evaluación y acreditación de las instituciones superiores de formación docente. En http://www.google.com/url?sa=t\&rc $\mathrm{t}=\mathrm{j} \& \mathrm{q}=$ \&esrc $=\mathrm{s} \&$ source $=$ web\& $\mathrm{cd}=2 \& \mathrm{ved}=0 \mathrm{CCQQFjAB} \& \mathrm{url}=\mathrm{http} \% 3 \mathrm{~A} \% 2 \mathrm{~F} \% 2 \mathrm{Fwww}$. minedu.gob.pe\%2FDeInteres\%2Fxtras\%2Fdownload.php\%3Flink\%3Dconeaces_estandares presentacion.pdf\&ei $=$ rO26VNCINIGkgwTuuIGABg\&usg $=$ AFQjCNHOEqkhVblddt-HXVHuC9pQeinAQ\&bvm = bv.83829542,d.eXY.

Consejo de Evaluación, Acreditación y Certificación de la Calidad de la Educación Superior No Universitaria. (2011) Reporte de gestión: Febrero - 2008 julio - 2011.

Consejo de Evaluación, Acreditación y Certificación de la Calidad de la Educación Superior No Universitaria. (2012) Taller de Capacitación con Miembros de Comités de Calidad de los Institutos y Escuelas de Educación Superior.: Sede Ica. En http://es.slideshare.net/coneacessineace/ acreditacion-sede-ica-noviembre-2012

Consejo de Evaluación, Acreditación y Certificación de la Calidad de la Educación Superior No Universitaria. (2013a) CONEACES: Rumbo a la Acreditación: Capacitarán a Institutos y Escuelas de Educación Superior en regiones. En http://www.sineace.gob.pe/capacitaran-iest-iesp-enregiones-para-acreditarse/

Consejo de Evaluación, Acreditación y Certificación de la Calidad de la Educación Superior No Universitaria. (2013b) Cinco años promoviendo la calidad de los institutos y escuelas de educación superior. En http://boletinsineace.blogspot.com/2013_01_01_archive.html.

De la Haya, R. (2005) Estudio sobre la situación de la educación superior tecnológica en el Perú. Lima, Perú: IESALC-UNESCO.

Fernández, N. (s. f.) La educación superior en América Latina y el Caribe y la evaluación y acreditación de su calidad: Situación, problemas y perspectivas. Buenos Aires, Argentina: Sociedad Andina de Estudios Comparados.

Instituto Peruano de Evaluación, Acreditación y Certificación de la Calidad de la Educación Básica. (2011) Dos décadas de formación profesional y certificación y certificación de competencias: Perú $1990-2010$.

Instituto Superior Tecnológico TECSUP (2014) Acreditaciones. En http://www.tecsup.edu.pe/ home/postulantes/acreditaciones/ 
Jaramillo, M., Valdivia, N. y Valenzuela, J, (2007) Políticas de capacitación ene le Perú: El rol de los actores nacionales e internacionales. Lima, Perú: Grupo de Análisis para el Desarrollo.

Jiménez, R. (2014) Ejército: "Acreditación de nuestras carreras es un hecho trascendente e histórico". En http://www.sineace.gob.pe/ejercito-acreditacion-de-nuestras-carreras-es-un-hechotrascendente-e-historico/

Ministerio de Educación (MINEDU) y Consejo Nacional de Educación (CNE). (2007) Proyecto Educativo Nacional al 2021 del Perú. Lima: CNE.

Morgan, P. (2014) La acreditación garantiza el derecho a una educación de calidad. http://www. sineace.gob.pe/la-acreditacion-garantiza-el-derecho-a-una-educacion-de-calidad/.

RPP Noticias. Solo tres institutos superiores han certificado su calidad educativa. (2013, 6 de mayo) RPP Noticias. En http://www.rpp.com.pe/2013-05-06-solo-tres-institutos-superiores-hancertificado-su-calidad-educativa-noticia_592232.html

Sistema Nacional de Evaluación, Acreditación y Certificación de la Calidad Educativa. (2011) Estudio cualitativo sobre atributos y determinantes de la calidad de la educación superior. En http://procalidad.gob.pe/documentos/tabla/Informe_final.pdf

Sistema Nacional de Evaluación, Acreditación y Certificación de la Calidad Educativa. (2014a) Más de 2,500 comités de calidad de instituciones educativas avanzan rumbo a la Acreditación. En http://www.sineace.gob.pe/mas-de-2500-comites-de-calidad-de-instituciones-educativasavanzan-rumbo-la-acreditacion/

Sistema Nacional de Evaluación, Acreditación y Certificación de la Calidad Educativa. (2014) Procedimiento para la evaluación y acreditación de las instituciones de educación superior no universitaria: Versión II. En http:/www.sineace.gob.pe/wp-content/uploads/2014/10/ ProcedimientoAcreditacion_IEES.pdf

Stiegler, K. (2014) Toulouse Lautrec recibe reconocimiento de CONEACES. JC Magacine. En http://www.jcmagazine.com/toulouse-lautrec-recibe-reconocimiento-de-coneaces/

Ybáñez, N. (2013) Instituto Continental. En https://mbasic.facebook.com/instituto.continental?v $=$ timeline\&timecutoff $=1392762275 \&$ page $=15 \&$ sectionLoading $\mathrm{ID}=\mathrm{m}$ timeline_loading_div_1 388563199_1357027200_8_15\&timeend $=1388563199 \&$ timestart $=13 \overline{5} 702720 \overline{0} \& \mathrm{tm}=\mathrm{AQD} \overline{\mathrm{kN}}$ TCBsWjMCIVN 